\title{
Platform oriented semantic description of pattern-based learning scenarios
}

\author{
Zeyneb Tadjine ${ }^{1}$, Lahcen Oubahssi ${ }^{1}$, Claudine Piau-Toffolon ${ }^{1}$, Sébastien Iksal $^{1}$ \\ 1LUNAM University, University of Maine, EA 4023, LIUM, 72085 Le Mans, France \\ \{FirstName.LastName\} @univ-lemans.fr
}

\begin{abstract}
In our research work, we address the issue of representing the learning scenario's concepts, in a learning platform. In this context, we have proposed a process for operationalizing pattern-based learning scenarios. We present the first two steps dealing with the new challenge of modeling deployable e-learning scenarios using Semantic Web technologies. It is primarily an ontology-based description of learning scenarios, which helps reducing the gap between human-readable and machine-readable vocabulary. We highlight the effectiveness of orienting teachers-designers, non-platform experts, toward creating adaptable and deployable learning scenarios. We defend that an assisted and platform-oriented design, allows the teachers to have a better pedagogical use of the embedded tools and features of learning platforms.
\end{abstract}

Keywords: Learning Design, learning platforms, operationalization, learning scenarios, patterns.

\section{$1 \quad$ Aim and Motivation}

Learning Management Systems (LMS) are more and more used by teachers, their use is not anymore restricted to content repository for distant learning [1] [2]. Nevertheless, we note that teachers find some difficulties using the LMS, especially when they are not platforms experts. The challenge is to easily master the process from the design to the operationalization of learning scenarios, for that, we believe that the operationalization of learning scenarios on LMSs is more than a technology-related question. Different research issues around instructional design are to be addressed in order to provide pedagogical expressiveness of the different elements within a learning scenario, while the design respects sufficiently the structure to describe the learning scenario [3] [4]. In our research, we seek to provide solutions to the problem of the automatic deployment of learning scenarios. We propose a process of the operationalization of pattern based learning scenarios [4]. The aim is to offer the use of a pattern formalism to create and edit learning scenarios, allowing the learning scenario design to be open enough to express teachers' concerns on one side and on the other side, structured enough to be machine interpretable for deployment purposes. In this 
paper we intend to mainly present a semantic model to help creating learning scenarios as a part of our process.

\section{Structuring and Indexing Platform-Based Learning Scenarios}

In order to define a clear idea of the way to address the challenge of properly design platform-oriented learning scenarios, we investigated the benefits, as well as the issues, regarding using a pattern-based LD tool by teachers-designers [2]. We studied the learning scenario from two viewpoints: (i) Starting from teachers intentions going down to its representation on an LMS, (ii) using a pattern-based design. This study allowed us to identify the assets (patterns formalism requirements and ontological modeling) leading us to automate the deployment of learning scenarios. As a result we have settled on semantically modeling and mapping the double vision: human intentions and platform representations for guaranteeing to teachers-designers a design tool able to assist them in deploying their learning scenarios with less effort of manual adaptations. We also proposed a classification of the different approaches dealing with learning design [5] [6] [7], more specifically, those using ontologies as a semantic base to improve the learning process [8] [9] [10] [11]. Although, all the effort made in developing systems to support the learning design process, literature has shown they had not yet reached a sufficient spread among teachers. We have noticed that most of the proposed design languages and tools do not preserve the semantic meaning of teachers' intention while transposing it on a LMS.

After that, we started collecting and structuring the available information and concepts related to the field of education [12] [13] [14] [15] [7]. We were concerned only by the learning scenario's concepts necessary to its deployment, justified by the fact that we focus our research on platform-oriented learning scenarios. This step is very important, since it is a key solution to index learning platforms pedagogical language into a general semantic description of a platform-oriented learning scenario. Studying the existing learning design repositories and theories where instructional scenarios can be modeled, we defined a five levels structure of the learning scenario, which represents the structuring step of our process. We believe that the right set of abstractions will give more benefits to easily map the human design language to the machine interpretable one. We had to make sure that the technological tools will easily support our proposed model. For that, the other point was to study an example of a deployed learning scenario. It consists in peer assessment of a synthesis. The course covers most of the features that Moodle 2.4 includes. Next, we explain an extract of concepts of our structure. We managed to introduce the most relevant concepts to deployment goals. The first level formalizes the notion of "learning scenario" in terms of structure and content, based on the different definitions researchers assigned to learning scenario [14]. A scenario describes roles, activities and also knowledge resources, tools and services necessary to the implementation of each activity. From all this emerges the most used concepts that summarizes the essence of a deployable learning scenario: the learning scenario structure is what defines in a design any sequential ordering of activities, it is mainly inspired from [14] research work, it is defined by a set of three concepts: "Structuration unit", "Activity sequence" and finally, "Elementary activity". This model was implemented as an ontology-based e-learning 
scenario model, using the Protégé tool ${ }^{1}$. Besides increasing the level of sharing content between teachers-designers, the ontological description will help us to ensure the support of the technological aspect for a learning scenario. Ontology will help teachers-designers to formalize pattern-based scenarios with the editing tool conformed to the conceptual framework we proposed.

The concept of "Elementary activity" is assigned to a category from bloom's taxonomy [13], the categorisation will help the teacher to better create pedagogically reusable pattern-based learning scenarios, as well as it will help us to index it according to the most suitable platform tool. Any learning scenario has some necessary conditions and rules to be executed as teachers-designers intended, and since our learning scenarios are designed to be platform oriented in terms of design and deployment, we must take into account both the platforms and the pedagogical point of view. For that, we defined two sets of constraints. The first one concerns the human reasoning of the right conditions to manage the learning scenario, as for example the fact of restricting the access of an activity to the learners on the base of the previous activities results. The second set of constraints concerns the machine readable part of the scenario, although the previous ones are also machine interpretable, but they mostly relate to a pedagogical use, while the platform oriented set is fully built on computed learning environments. As we studied Moodle 2.4 platform, we retained the constraints adding a pedagogical dimension to the deployed scenario. We take the visibility constraint as an example, this added value allows the teacher to hide any activity for the learner until a time he judged suitable for his goals: it could be according to a score of a given evaluation, or a certain duration in time etc. We complete pedagogical goals and all others concepts describing evaluation in a learning scenario by all missing informations needed to operationalize an evaluation based scenario. The agent of evaluation could be the teacher, the students, in case of a peer assessment, and even the learning platform itself in case of an auto evaluation. We also note that an evaluation activity is a set of some evaluation tools, helping the teacher to assess students according to their needs: graded assessment, auto-evaluation, paper exam, quizz, with or without feedback etc.

After identifying our structure, helping teachers towards a platform-oriented learning design, we must ensure the mechanism to automatically transform their pedagogical intentions into modules and content on the targeted learning platform. Next, we show through an example the way we deducted our manual ontology alignments between the semantic descriptions of our pattern-based learning scenario and Moodle's pedagogical embedded language. We started by transforming the metamodel into a semantic description; this is a very important phase because it is the first step toward a platform semantic description as a form of an ontology. In order to align our two semantic descriptions, we studied the example presented earlier about peer assessment in Moodle, adding to that our collaboration with a pedagogical designer, to come up with the right mappings of Moodle's tools and features. Starting from the most frequently functions required by teachers-designers, we grouped the set of offered tools as follows: collaborative work tools (glossary, journal, wiki, workshop etc.), synchro-

\footnotetext{
${ }^{1}$ http://protege.stanford.edu/
} 
nous and asynchronous communicative tools work tools (forum, chat, and survey), learning tools (lesson) and evaluative tools (assignments, workshop, quizz, etc.). We believe that this work has to be refined with teachers' experiences using learning platforms, thus we highlight again the importance of using a semantic description because it is extensible, and allows indexing and adding of more features as the technological updates are evolving around distance learning.

\section{References}

1. El Mawas, N., Oubahssi, L., \& Laforcade, P. Making explicit the Moodle instructional design language. In Advanced Learning Technologies (ICALT), 2015 IEEE 15th International Conference on (pp. 185-189). IEEE. (2015, July).

2. Oubahssi, L., Piau-Toffolon, C., Clayer, J. P., \& El Mawas, N. Design and Operationalization of Learning Situations Based on Patterns for a Public in Professional Development. In Open Learning and Teaching in Educational Communities (pp. 580-581). Springer International Publishing. (2014).

3. Prieto, L. P., Asensio-Pérez, J. I., Dimitriadis, Y., Gómez-Sánchez, E., \& Muñoz-Cristóbal, J. A. GLUE!-PS: a multi-language architecture and data model to deploy TEL designs to multiple learning environments. In Towards ubiquitous learning (pp. 285-298). Springer Berlin Heidelberg. (2011).

4. Tadjine, Z., Oubahssi, L., Piau-Toffolon, C., \& Iksal, S. A Process Using Ontology to Automate the Operationalization of Pattern-Based Learning Scenarios. In Computer Supported Education (pp. 444-461). Springer International Publishing. (2015).

5. Laurillard, D. Teaching as a design science: Building pedagogical patterns for learning and technology. Routledge. (2013).

6. Koper, R. Current research in learning design. Educational Technology \& Society, 9(1), 1322. (2006).

7. Koper, R. Modeling Units of Study from a Pedagogical Perspective: the pedagogical metamodel behind EML. Heerlen, Open Universiteit Nederland En ligne.(2001).

8. Chimalakonda, S., \& Nori, K. V. A Patterns-Based Approach for Modeling Instructional Design and TEL Systems. In Advanced Learning Technologies (ICALT), 2014 IEEE 14th International Conference on (pp. 54-56). IEEE. (2014, July).

9. Fensel, D. Ontologies (pp. 11-18). Springer Berlin Heidelberg. (2001).

10. Amorim, R. R., Lama, M., Sánchez, E., Riera, A., \& Vila, X. A. A learning design ontology based on the IMS specification. Educational Technology \& Society, 9(1), 38-57. (2006).

11. Mizoguchi, R., \& Bourdeau, J. Using ontological engineering to overcome common AI-ED problems. Journal of Artificial Intelligence and Education, 11, 107-121. (2000).

12. Paquette, G. A Competency-Based Ontology for Learning Design Repositories. International Journal of Advanced Computer Science and Applications, 5(1), 55-62. (2014).

13. Krathwohl, D. R. A revision of Bloom's taxonomy: An overview. Theory into practice, 41(4), 212-218. (2002).

14. Lejeune, A., \& Pernin, J. P. A Taxonomy for Scenario-Based Engineering. In CELDA (pp. 249-256). (2004, December).

15. Churchill, D. Towards a useful classification of learning objects. Educational Technology Research and Development, 55(5), 479-497. (2007). 